\title{
Subconjunctival Use of Allogeneic Mesenchymal Stem Cells to Treat Chronic Superficial Keratitis in German Shepherd Dogs: Pilot Study.
}

\author{
Alexandre Luiz Pereira ( $\nabla$ vet.arcadenoe@hotmail.com ) \\ Maura Wanderley Bittencourt \\ Universidade Estadual de Campinas \\ Michele Andrade Barros \\ Pontificia Universidade Catolica de Campinas \\ Rodolfo Malago \\ Universidade Estadual de Campinas \\ João Flávio Panattoni Martins \\ Pontificia Universidade Catolica de Campinas

\section{Bruna Morais} \\ Pontificia Universidade Catolica de Campinas

\section{Fabiano Montiani-Ferreira} \\ Universidade Federal do Parana \\ Jose Paulo Cabral de Vasconcellos \\ Universidade Estadual de Campinas
}

Universidade Estadual de Campinas https://orcid.org/0000-0002-5057-0365

\section{Research article}

Keywords: Chronic Superficial Keratitis, Regenerative medicine, Autoimmune disease, Mesenchymal stem cells, Cornea, Dog

Posted Date: September 3rd, 2020

DOI: https://doi.org/10.21203/rs.3.rs-51716/v1

License: (c) (i) This work is licensed under a Creative Commons Attribution 4.0 International License. Read Full License

Version of Record: A version of this preprint was published at Open Veterinary Journal on October 7th, 2022. See the published version at https://doi.org/10.5455/OVJ.2022.v12.i5.20. 


\section{Abstract}

Background: Chronic superficial keratitis (CSK) is an ocular condition in dogs characterized by corneal opacification leading to visual function impairment. The aim of this study was to evaluate the clinical effect of allogeneic mesenchymal stem cells (MSC) of adipose tissue in dogs with CSK.

Results: The conventional treatment group received topical Prednisolone $1 \%$ and the experimental group received MSC on the order of $1 \times 10^{6}$ were injected via subconjunctival in the peri-limbal region at 0 and 30 days. The animals were followed for 110 days to monitor the clinical progression and the statistical analysis was performed. Comparison of initial and final images revealed that areas with neovascularization and inflammatory infiltrate regressed in most eyes in both the conventional and experimental groups at the end of the 110 days. The experimental group responded positively to MSC treatment, but bellow the response achieved by the conventional group. Some areas of opacity of the cornea become transparent after transplantation of MSC. No local or systemic side effects were observed.

Conclusions: Stem cells treatment produced clinical improvement in patients with CSK after 110 days without use of any drug combination and any local or systemic side effects, even if the improvement obtained was less than that seen with conventional corticosteroid therapy.

\section{Background}

Chronic superficial keratitis (CSK) or Uberreiter Syndrome (1)represent a chronic, inflammatory non-ulcerative disease of the superficial corneal stroma, which causes significant damage and consequent visual impairment in dogs (2). This condition is particularly common in German shepherd and Greyhounds, Belgian Shepherd dogs, but other breeds may be affected $(3,4)$. The disease also affects humans $(5)$ and affected dogs may serve as a model species for this disease. The CSK diagnoses are usually made through typical clinical appearance and breed association. Clinical signs of the disease are conjunctival redness and corneal pigmentation bilaterally. Corneal lesion development, usually in its ventrolateral region, has a typical pattern of cellular infiltration from the limbus into the cornea as well as the conjunctiva adjacent to the cornea affected region presents signs of inflammation. In the early stages of CSK, epithelial cells proliferate and the superficial stroma is infiltrated by plasma cells and lymphocytes (6). As the disease progresses, melanocytes, histiocytes and fibrocytes enter the cornea, leading to edema and neovascularization. In the advanced stages, the epithelium and anterior stroma are heavily pigmented and vascularized. It has also been suggested that an autoimmune mechanism is involved in the pathophysiology of this condition that shows a predominance of CD4+ lymphocytes in the infiltrate (7). The initial researchers suggested an environmental risk factors such as ultraviolet rays and high altitude for this condition, but more recent searches have shown a seric variation in Vascular endothelial growth factor concentration (8), and also a genetic basis for CSK (5,9-12).

Corticosteroids are used as anti-inflammatory and immunosuppressive agents and are generally accepted as a form of medical management for CSK(6). The needs of treatment throughout the lifetime exposures the patients to important side effects from corticosteroids. Thus, efforts are being made to develop alternative therapies to inhibit the immune response and chronic inflammatory processes in order to reduce the suffering of animals with CSK and the need for their long periods of care. Many experimental tests have been done with therapies including contact lenses(13), radiotherapy with surgical procedures(14), and immunosuppressive drugs(2,15-17). Recently, the use of tacrolimus and/or cyclosporine has been considered to be the therapy of choice.

MSCs are a type of multipotent cell originally isolated from bone marrow $(18,19)$ that have subsequently been isolated from other tissues(20); such as adipose tissue(21,22). Recently, MSCs are a therapeutic approach with have been performed to treat ophthalmologic diseases in dogs with keratoconjunctivitis sicca (KCS) $(23,24)$ and eosinofilic keratitis in cats(25). In these studies, MSC transplantation was effective in reducing inflammation of the lacrimal gland in dogs and 
recovering normal lacrimal production and controlling corneal infiltration in cats. Additionally, there are many studies in animal models of corneal diseases showing the ability of these cells to prevent scar formation as well as influence and modulate excessive immune response (26-28). This potent action of MSC as regulators of the immune response is related to release of soluble bioactive factors PGE2, IL-1, IL-4, IL-6, TGF- $\beta$ and IDO in tissue(29-31).

The cornea is an immune privileged tissue (32) and its anatomical characteristics allow easy treatment assessment and evaluation of the therapeutic efficacy in live animals after MSC transplantation. Additionally, dogs share corneal characteristics with humans leading to translate basic mechanistic models opening new potential therapeutic approaches in humans. In another hand, the advances in animal models could be applied directly in clinical veterinary medicine as well.

This study aims to evaluate the treatment of CSK in dogs using subconjunctival allogeneic MSC transplantation. At same time, the short-term safety of subconjunctival transplantation of allogeneic MSCs will be assayed.This study, therefore sought, first, to investigate and describe the initial results of MSC therapy in dogs with CSK without the concomitant use of conventional drugs therapy and in second, to compare with the conventional treatment with corticosteroids.

\section{Results}

\subsection{Safety of Allogeneic MSC Transplantation}

During the ophthalmologic and clinical evaluation, they were not observed any side effects, such as ocular pain, discomfort or irritation of the conjunctival tissue secondary to MSC transplantation. At the time of follow-up, the ophthalmologic examination showed no changes in tear production, corneal epithelial integrity, and intraocular pressure, as suggested by the results of the Schirmer test, fluorescein test and tonometry that maintained all of them within the range of normality at all moments of evaluation. As some eyes favorably changed the clinical manifestation of neovascularization, the Menace response test became more pronounced confirming the improvement in visual ability. In addition, no changes were detected in appetite, defecation/urination frequency, body weight or body temperature and no allergic reaction was observed. Participants remained healthy throughout follow-up, showing that treatment did not produce detectable systemic changes. The owners' adherence proved to be sufficient and efficient, as evidenced by the frequency of clinical returns for evaluations and second applications of MSC, as well as by the reported descriptions of the subjective values of pain and ocular discomfort and descriptions of the daily behavior patterns of the animals.

\subsection{Laboratory Evaluation}

Corneal cytology revealed no signs of neoplasia. Instead, the epithelial phenotype resulting from this ocular surface evaluation demonstrated noncorneal tissue, evidenced by the presence of goblet cells commonly found in the conjunctiva 4 (data not shown).

\subsection{Description of clinical changes in the ocular surface of the experimental group:}

Participant \# 1 at baseline had neovascularization migrating centripetally at 360 degrees resulting in opacity that blocked anterior chamber visibility along with poor visual ability behavior evidenced by the negative result in the menace response test. At 30 days after the first MSC transplant, a reduction of these blood vessels allowed a partial clinical examination of the anterior chamber as well as the improvement of the menace response test. The opacity remained in the center of the cornea while the pigmentation was more concentrated in the periphery. Between the second MSC transplant until the end of the days of follow-up, neovascularization continued to decline to the point where only a small number of blood vessels

Page 3/18 
were visible, and the periphery of the corneas became more transparent, while in the left eye pigmentation cicatricial was advancing towards the center of the cornea. At the end of the 110-day follow-up, visual behavior was clinically normal (Figure 1. $A$ and $B$ )

Participant \# 2 showed the lateral portion of the affected corneas, occupying 3 quadrants of the area of the corneas. The visual capacity and behavior were normal. After 30 days, the right eye demonstrated a reduction of neovascularization, however, in the left eye, the blood vessels increased and covered the entire cornea. This was the only eye in this study in which a clinical worsening pattern was observed. At the end of the follow-up, the right cornea showed no blood vessel and the pigmentation clinically occupied the same area as the initial blood vessels. The left eye also showed the reduction of neovascularization to a lesser extent than observed on day 0 , but the pigmentation area was higher. The visual behavior remained clinically normal (Figure 1.C and D)

Participant \# 3 had his right eye with the greatest neovascularization and was clinically blind. The left eye showed advanced pigmentation with vessels but still had a positive menace response test. At the end of the follow-up, clinical appearance in the right eye showed a reduction in the vessels and presented a positive menace response test, while the left eye showed reduced pigmentation intensity

Participant \# 4 had the disease at the initial stage with only 1 quadrant affected in both eyes. During follow-up, vessels and opacity were progressively reduced, the cornea became clear with little pigmentation reaction

\subsection{Description of clinical changes in the ocular surface of the conventional group}

The participant \#5 presented the disease at the initial stage with only 1 quadrant affected in the right eye and 2 quadrants in the left eye. The intermediate data were not obtained. At 110 days in both eyes, the inflammatory process had regressed, but the pigmented areas were larger than the initially affected areas

Participant \#6 Similarly, he had the disease at the initial stage with only 2 lower quadrants affected in both eyes. At 30 days of follow-up, regression of the inflammatory process and pigmentation in the same regions initially affected in the corneas was noticeable. At 110 days in both eyes the pigmented area had regressed in area and density (Figure 2.A and B)

Participant \#7 had the disease in a more advanced stage with a chronic pattern due to the presence of an extensive visible pigmentation, in both corneas affected. At 30 days of follow-up, there was already a marked decrease in

neovascularization and the pigmentation expanded a little more, without compromising all regions initially affected in the corneas. At 110 days in both eyes, the pigmented area had regressed in area and density, but the cornea showed a reappearance of neovascularization. The owner reported that he had stopped the daily medication (Figure 2.C and D)

Participant \#8 had the disease in the initial clinical stage, with only 2 quadrants affected in the right eye and 1 quadrant in the left eye and with pigmentation visible only near the limbus. At 30 days of follow-up, there was complete remission of the active inflammation process, with a marked decrease in neovascularization, and pigmentation expanded somewhat further from the initial position without compromising all regions initially affected in the corneas. At 110 days in both eyes, the pigmented area had regressed in area and density leaving a little scarring reaction

\subsection{Evaluation of the ocular surface:}

The mean values of the examiners for the experimental group are expressed in Table 1 and the conventional treatment group in Table 2 (after References). 
There was no statistically significant difference between the readings of ocular surface alterations among the examiners $(p=0.5474)$

A comparison between the initial data of the ocular surface changes of the two groups was performed to verify the similarity relationship between them, and there was no significant difference on day $0(p=0.2786)$ (Figure 3.A).

Intra-group evaluation

The experimental group presented the following observations:

- For affected areas, there was no significant difference $(p=0.25)$ comparing the initial (day 0$)$ and intermediate (day 30$)$ days of the inflamed areas. But it reached a significant difference $(p=0.0078)$ comparing the initial and last follow-up (day 110 ) with the reduction of the area with active inflammation in $8 / 8$ eyes (Figure 4 . A). Whereas for pigmented areas there was no significant difference $(p=0.25)$ comparing the initial and final days (Figure 4 . B)

The conventional treatment group presented the following observations:

- For affected areas, there was a significant difference $(p=0.0313)$ comparing the initial and intermediate days (without considering participant $A$ that did not have paired data), with a reduction in $6 / 6$ eyes; as well as there was a significant difference $(p=0.0391)$ comparing the initial and final evaluation with a reduction in $7 / 8$ eyes (Graph 2 . $C)$. Whereas for pigmented areas, there was no significant difference $(p=0.3828)$ comparing the initial and final days $(G r a p h 2$. D).

Evaluation among groups is observed:

- There was a significant difference $(p=0.0426)$ comparing the values of the intermediate day between the groups favorable to conventional group (Figure 3 . B) and this difference remained significant $(p=0.0263$ ) comparing the final day values between groups for affected areas (Figure 3. C).

Table 1

Experimental group. Quantitative measures of the affected and pigmented areas and qualitative measures of density and area.

\begin{tabular}{|c|c|c|c|c|c|c|c|c|c|c|c|}
\hline \multirow[b]{2}{*}{ Dog } & \multirow[b]{2}{*}{ Age } & \multirow[b]{2}{*}{ Eye } & \multicolumn{3}{|c|}{$\begin{array}{l}\text { Corneal vascularization(score of } \\
\text { intensity and number of affected } \\
\text { quadrants) }\end{array}$} & \multicolumn{3}{|c|}{$\begin{array}{l}\text { Vascular area in the } \\
\text { cornea }(\%)\end{array}$} & \multicolumn{3}{|c|}{$\begin{array}{l}\text { Pigmented area on the } \\
\text { cornea (\%) }\end{array}$} \\
\hline & & & $\begin{array}{l}\text { Before } \\
\text { treatment }\end{array}$ & Day 30 & $\begin{array}{l}\text { Day } \\
110\end{array}$ & $\begin{array}{l}\text { Before } \\
\text { treatment }\end{array}$ & $\begin{array}{l}\text { Day } \\
30^{\mathrm{a}}\end{array}$ & $\begin{array}{l}\text { Day } \\
110^{\mathrm{b}}\end{array}$ & $\begin{array}{l}\text { Before } \\
\text { treatment }\end{array}$ & $\begin{array}{l}\text { Day } \\
30^{c}\end{array}$ & $\begin{array}{l}\text { Day } \\
110^{\mathrm{C}}\end{array}$ \\
\hline \multirow[t]{2}{*}{1} & 5 & $\mathrm{RE}$ & $++++/ 4$ & $++/ 3$ & $++/ 3$ & 95.20 & 89.31 & 91.03 & 12.64 & 26.39 & 14.11 \\
\hline & & LE & $++++/ 4$ & $++/ 3$ & $++/ 3$ & 93.29 & 87.46 & 77.05 & 10.27 & 18.82 & 52.29 \\
\hline \multirow[t]{2}{*}{2} & 4 & $\mathrm{RE}$ & $++++/ 3$ & $++/ 2$ & $-/ 2$ & 69.93 & 61.18 & 33.63 & 8.73 & 38.87 & 36.36 \\
\hline & & LE & $+++/ 3$ & $\begin{array}{l}++++ \\
/ 4\end{array}$ & $+/ 4$ & 75.01 & 87.53 & 45.81 & 21.36 & 26.38 & 40.92 \\
\hline \multirow[t]{2}{*}{3} & 10 & $\mathrm{RE}$ & $++++/ 4$ & $\begin{array}{l}++++ \\
/ 4\end{array}$ & $\begin{array}{l}++++ \\
/ 2\end{array}$ & 95.65 & 95.83 & 83.62 & 7.74 & 8.74 & 8.75 \\
\hline & & LE & $+++/ 3$ & $\begin{array}{l}+++/ \\
4\end{array}$ & $\begin{array}{l}+++/ \\
4\end{array}$ & 89.95 & 82.84 & 88.81 & 82.91 & 56.97 & 63.36 \\
\hline \multirow[t]{2}{*}{4} & 4 & $\mathrm{RE}$ & $+++/ 1$ & $\begin{array}{l}+++/ \\
1\end{array}$ & $+/ 1$ & 30.65 & 27.19 & 24.26 & 12.7 & 12.10 & 10.39 \\
\hline & & LE & $++/ 1$ & $+/ 1$ & $+/ 1$ & 31.27 & 23.81 & 20.93 & 0.74 & 0.87 & 1.59 \\
\hline
\end{tabular}


Age = years. RE = Right eye; LE = Left eye. Score "+" = low density "++++" = high density. ${ }^{\text {a.b.c }}=$ statistical groups.

Table 2

Conventional group. Quantitative measures of the affected and pigmented areas and qualitative measures of density and area

\begin{tabular}{|c|c|c|c|c|c|c|c|c|c|c|c|}
\hline \multirow[b]{2}{*}{ Dog } & \multirow[b]{2}{*}{ Age } & \multirow[b]{2}{*}{ Eye } & \multicolumn{3}{|c|}{$\begin{array}{l}\text { Corneal } \\
\text { vascularization(score of } \\
\text { intensity and number of } \\
\text { affected quadrants) }\end{array}$} & \multicolumn{3}{|c|}{ Vascular area in the cornea(\%) } & \multicolumn{3}{|c|}{$\begin{array}{l}\text { Pigmented area on the cornea } \\
(\%)\end{array}$} \\
\hline & & & $\begin{array}{l}\text { Before } \\
\text { treatment }\end{array}$ & $\begin{array}{l}\text { Day } \\
30\end{array}$ & $\begin{array}{l}\text { Day } \\
110\end{array}$ & $\begin{array}{l}\text { Before } \\
\text { treatment }^{a}\end{array}$ & Day $30^{b}$ & $\begin{array}{l}\text { Day } \\
110^{c}\end{array}$ & $\begin{array}{l}\text { Before } \\
\text { treatment }^{d}\end{array}$ & Day $30^{d}$ & $\begin{array}{l}\text { Day } \\
110^{d}\end{array}$ \\
\hline \multirow[t]{2}{*}{5} & \multirow[t]{2}{*}{5} & $\mathrm{RE}$ & $+++/ 1$ & * & $+/ 2$ & 42.61 & * & 57.94 & 16.91 & * & 43.10 \\
\hline & & LE & $++/ 3$ & * & $+/ 3$ & 56.34 & * & 42.06 & 14.13 & * & 50.85 \\
\hline \multirow[t]{2}{*}{6} & \multirow[t]{2}{*}{4} & $\mathrm{RE}$ & $+/ 3$ & $+/ 2$ & $++/ 2$ & 64.24 & 46.04 & 15.93 & 29.28 & 35.10 & 38.94 \\
\hline & & LE & $+/ 2$ & $+/ 2$ & $+/ 1$ & 47.14 & 35.80 & 15.93 & 11.22 & 27.1 & 22.89 \\
\hline \multirow[t]{2}{*}{7} & \multirow[t]{2}{*}{10} & $\mathrm{RE}$ & $++++/ 4$ & $+/ 2$ & $++/ 3$ & 90.43 & 34.49 & 14.59 & 36.02 & 58.17 & 23.29 \\
\hline & & LE & $++++/ 4$ & $+/ 3$ & $+/ 3$ & 80.68 & 53.59 & 66.46 & 29.01 & 40.26 & 37.17 \\
\hline \multirow[t]{2}{*}{8} & \multirow[t]{2}{*}{4} & $\mathrm{RE}$ & $++++/ 2$ & $-/-$ & $-/-$ & 34.37 & 11.12 & 7.49 & 9.97 & 13.79 & 8.09 \\
\hline & & LE & $++++/ 1$ & $-/-$ & $-/-$ & 37.99 & 13.20 & 6.35 & 17.65 & 9.75 & 7.58 \\
\hline
\end{tabular}

Age = years. RE = Right eye; LE= Left eye. Score "-" = normal, "+" = low density, "++++" = high density. a.b.c,d= statistical groups." *"=absent

\section{Discussion}

This study, therefore sought first, to investigate and describe the initial results of MSC therapy in dogs with CSK without the concomitant use of conventional topical immunomodulating therapy and in second, to compare with the conventional treatment with corticosteroids similar to other ophthalmic diseases considered autoimmune that were also treated with corticosteroids, such as plasmacytic conjunctivitis (33), eosinophilic keratoconjuntivitis(34) and nodular granulomatous episcleritis(35). In this pilot study of a series of cases of dogs being treated with MSC transplantation, it was suggested the safe of its use through subconjunctival perilimbal application in dogs naturally affected with CSK. There was a significant improvement in neovascularization in the experimental group, as well as in clinical visual tests and visual behavior, according to the owner's reports. Considering that in this pathology there is no spontaneous regression of inflammation, the results show that the MSCs were able to reduce neovascularization, and in fact, the understanding of these actions requires new studies to elucidate which chemotactic mechanisms have changed in the inflammatory responses and the infiltration of cells of the immune system. After 110 days of follow-up, the reduction in the affected areas in relation to neovascularization varied up to $36 \%$ of the total corneal area and corresponded to the mean values obtained in several studies using conventional therapies. In the literature, currently, the clinical response with these drugs is satisfactory, but the daily use may interfere with adherence to treatment (17). Several studies have used different products applied daily, including cyclosporin A and dexamethasone (15), pimecrolimus (16), tacrolimus with (2) and without(17) the combination of Dimethyl sulfoxide (DMSO) and often lead to a reduction of neovascularization and corneal opacities within 4 to 6 weeks. Only pimecrolimus presented reduction of cicatricial pigmentation(16). However, in discontinuing such medications used to control the disease, the pathological condition relapses in a short time(15). Other approaches described include that suggested by Allgoewer and Hoecht (2010), who using radiotherapy in combination with superficial keratectomy, demonstrated that the reduction in pigmentation and vascularization was maintained over the 24-week observation period. 
The use of contact lenses with ultraviolet radiation blocking has been shown to be a protocol that does not produce the desired therapeutic effect (13).

One of the advantages that stands out in MSC therapy, and is important for the owners' accession is that daily topical administration of medications was not necessary. The animals did not experience any eye discomfort or any other reaction that the owners could observe and report. The fact that fluorescein, Schirmer and tonometry tests were normal indicating that there were no adverse reactions in ocular surface and that subconjunctival administration of MSCs did not affect the anatomical and physiological processes associated with these clinical trials.

In the experimental group there was no statistical variation in neovascularization between days 0 and 30 . One possible explanation would be that the first transplantation of stem cells alone failed to produce a significant result. However, after the second application (at $30^{\text {th }}$ day) the clinical improvement can be noted. As for pigmentation, in the other studies for CSK it is observed that there is no direct correlation between protocol of application and the result obtained, so that there are always patients with the reduction of pigmentation while in others there is growth or variation in pigment density ${ }^{2}$. In our experiment, the use of stem cells, in the model studied, did not produce consistent clinical change of corneal pigmentation, similarly to the descriptions in the literature. Areas with pigmentation increased in varying degrees in six eyes and reduced in two eyes. The pigmentation would probably be camouflaged by inflammation and only became visible once the vascularization had receded ${ }^{6}$. It is also possible that stem cells do not affect pigment production, or that the pigmented tissue does not provide a suitable microenvironment for the immunomodulatory action of stem cells.

Statistical analysis of the experimental group demonstrates that there was a significant change in the ocular surface, confirming that the MSC in this protocol induced favorable clinical alterations at the end of follow up.

In spite of the recent research demonstrate, genetically, that overall homozygosity for the MHC class II region is associated with increased risk in CSK the etiology of CSK is still unknown $(11,36)$. Phenotypically, has been observed that although the disease occurs in the stromal layer of the cornea, the cytology of the corneal surface has indicated the presence of conjunctivalization of the cornea in all the patients with CSK, it is possible to suppose that these patients may have some type of impairment of the barrier function (37). Recent studies have shown the importance of limbus stem cells in preserving the specialized characteristics of the cornea. When there is a deficiency of limbus stem cells, the adjacent cornea becomes opaque as a result of conjunctivalization, with irregular appearance and presence of blood vessels and lymphocytic infiltrate (5). Chronic inflammatory conditions such as CSK may be associated with this deficiency. Although the subconjunctival administration of MSC has produced a clinical improvement in CSK in dogs, the process by which the MSC acts have not yet been elucidated. In parallel, despite the conventional group, having demonstrated that the antiinflammatory action of corticosteroids is more efficient in suppressing CSK and promoted the regression of neovascularization in a time similar to that described by Williams et al. (1995), the side effects of continued treatment are important and when the protocol is interrupted, the disease manifests itself again.

The result was more efficient in time response and remission intensity of the active inflammatory process when compared to the experimental group. However, it should be considered that the effects of MSCs could interact and regulate the corneal microenvironment bringing its results closer to the use of known drugs in a safe manner, without the need for continuous use of them.

Other studies should be conducted to investigate the beneficial and undesirable effects of this potential new treatment. To this end, the clinical stage of the disease, the number of cells administered, the number of applications and the interval between applications can be better determined and standardized in order to obtain even greater control of the therapeutic response. These variations may be better defined if the paracrine mechanisms of MSC in the microenvironment of the ocular surface are elucidated. MSCs secrete interleukins with anti-inflammatory action, for example, interleukins IL-4, IL-10 and IL-13 and also the growth factor of hepatocytes (HGF), TGF-B and PGE2, which are capable of suppressing IFN- $\gamma$ 
production from Th11 cells. Investigating whether these mechanisms occurred in the eyes of the participating dogs is an important part of the next studies (38-40).

New challenges must therefore be overcome, including investigating the intrinsic mechanisms by which MSCs act by identifying the cytokines found on the surface of the eye at various stages of the disease and identifying the membrane receptors where these cytokines act, whether in the various cells involved in the immune response or in the native cells on the ocular surface.

Once this information is available, CSK and other ocular surface diseases may be better understood and it may even be possible for veterinarians to provide the optimal combination of treatment for each patient at each stage of the disease.

This study has limitations, mainly non-randomization between conventional treatment and MSC, although the groups were comparable in relation to the clinical parameters evaluated at the beginning of the study. Despite the small number of participants, this was sufficient to indicate a clinical improvement in both groups. The hypothesis of the mechanisms by which there was the clinical improvement with the use of MSC in CSK is beyond the scope of this study. In addition, if MSC treatment becomes feasible, it must be considered that the need for sedative procedures to administer MSC may be the only limiting factor for high-risk patients.

\section{Conclusion}

The subconjunctival use of adult allogeneic mesenchymal stem cells from adipose issue was clinically safe for use in dogs during the follow-up period. It promoted an improvement in the clinical manifestation of chronic superficial keratitis in naturally affected German shepherd dogs, as demonstrated by the reduction of the intensity and extension of the affected corneal areas without the use of any drug combination.

\section{Methods}

This is a prospective interventional case control study comprising a series of 8 cases of dogs with CSK diagnosis. Dogs were included in this study from May 2015 to May 2017 in Atibaia, São Paulo, Brazil are animals seen in the routine of a private veterinary clinic specializing in ophthalmology. All procedures with animals were conducted according to the guidelines approved by the Ethics Committee of Care and Animal Use of the State University of Campinas (UNICAMP), Brazil, under protocol number 3636-1. The owners of the animals signed the free and informed consent form.

The inclusion criteria were: a) the presence of clinical signs compatible with the description of the disease, which includes: the presence of chronic, non-ulcerative keratitis, mainly in the lower lateral limbus with a combination of neovascularization and pigmentation that extends to the center of the cornea. In some cases, a white line or white spots often appear on the clear cornea 1 to $2 \mathrm{~mm}$ before the blood vessels as well as thickening and pigmentation of the third eyelid may be present; b) the dogs must have the phenotypic characterization of the breed "German shepherd" as shown in officials sites of cynophilia as http://clubepastoralemao.com/index.php/a-raca/padrao-oficial-da-raca-pastor-alemao-fci;

c) patients were not receiving any prior medication. Exclusion Criteria were the presence of other ocular conditions, such as corneal ulceration, dry keratoconjunctivitis, glaucoma, infectious keratitis or conjunctivitis, and any other ocular or systemic diseases, including the presence of neoplasia. Serious adverse reactions that could compromise the animal's vision permanently or endanger his or her life were considered criteria for stopping the study. A total of 16 eyes of seven females and one male of the German shepherd breed, aged 4 to 10 years, and weight from 30 to $35 \mathrm{Kg}$, participated in this study. All recruited participants were not receiving conventional immunosuppressive treatment before and during the study period.

\subsection{Clinical Evaluation}


The dogs were undergone to systemic and ophthalmologic evaluations. The systemic evaluation included abdominal ultrasound and chest X-ray, complete blood count and serum biochemistry to exclude other systemic diseases. The ophthalmologic evaluation included a neuro-ophthalmological examination (dazzle reflex, photomotor pupillary reflex and menace response test), Schirmer I tear test (STT-I characterized by measurement without the use of topical anesthesia) (Schirmer Test, Ophthalmos ${ }^{\circledR}$, São Paulo), applanation tonometry (Tono-Pen, Reichert Inc ${ }^{\circledR}$, USA). ), fluorescein test (Fluorescein strips, Ophthalmos ${ }^{\circledR}$, São Paulo), slit lamp biomicroscopy (Kowa, Japan ${ }^{\circledR}$ ), indirect ophthalmoscopy (Ocular Instruments ${ }^{\circledR}$, USA) and corneoconjunctival cytology were also performed to identify different types of ocular surface cells to exclude neoplasia. The dogs were monitored clinically at days 0, 30 and 110 days after diagnosis and treatment. The day of the first transplant was considered as day 0.

Each evaluation comprised comprehensive clinical and ophthalmologic examination, including evaluation of the corneal surface with fluorescein, tonometry, and Schirmer's test. Normal values for the Schirmer Type I Test in dogs comprise measurements between $15 \mathrm{~mm}$ and $25 \mathrm{~mm}$ in 1 minute, and normal tonometry values are between $10 \mathrm{mmHg}$ and $25 \mathrm{mmHg}$.

The clinical manifestation of pain and ocular discomfort in dogs is characterized by an increase in the natural rhythm of the blinking to the point of keeping the eyelids closed (blepharospasm), associated with increased tear production.

Perception of vision in animals is based on ambulation tests in known and unknown environments in light and dark conditions, usually with alternating obstacles in the eyes (signaling cones and common site furniture) to confirm the ability to deviate from them. Also, called the Menace response test by the examiner is to cover one eye and make a movement of approaching the hand in front of the open eye without producing sound and displacement of air or touching the hair of the muzzle in order to observe the ability of the animal to blink and move the head in order to avoid the approaching aggression (this test associates the vision with the cognitive capacity of interpretation and several neurological pathways related to the cranial nerves). These signs were monitored on the days of follow-up and the owners were trained to observe and report the occurrence of variations of them at home.

The state of the cornea was also evaluated; a subjective scale was standardized in relation to its transparency from 0 to 4 , being 0 the equivalent of a normal and 4 totally opaque cornea and dividing the corneal surface into 4 quadrants, being evaluated how many quadrants were involved. The areas of corneal vascularization added to the areas with inflammatory infiltrate defined the measures of the active "affected area". The pigmented areas added separately comprise the areas with the disease in the inactive or scarring phase. To perform such objective measurements, photographs were taken at each evaluation time, at 0,30 and 110 days. To measure the photographs, we used free software on the internet, qualified for image analysis (ImageJ -http://imagej.nih.gov/ij/). The photographs were all taken by the same operator and produced under the same conditions, with the same lighting and equipment to ensure the consistency of the results. In the evaluation criteria in ImageJ, the total area of the cornea in a given image was defined as $100 \%$.

The injured area was a percentage of the total cornea, considered the sum of the neovascularized area and opacity area (inflammatory infiltrate), similar to that described by Balicki (2012). The pigmented area was measured separately because it was considered the scarring reaction of the disease

Ocular surface measurements were carried out in a blind way by three examiners working in the area of veterinary ophthalmology for more than 10 years, all trained at the same institution called ANCLIVEPA-SP (Association of Veterinary Clinicians of Small Animals -SP) and who did not know the protocol and the timing of each image.

\subsection{Inclusion in study groups}

The owners have received clinical instruction on the pathology of their companion animals, and available treatment models. They had free choice on which protocol they would participate: in the experimental model with MSC, or in the 
conventional treatment with topical anti-inflammatories. The choice of which group their dogs would be enrolled did not have the evaluator's participation. According to the clinical routine and the casuist, 2 groups were defined with 4 animals, 8 eyes in each:

- Experimental group: participants who received 2 applications of 1 million MSC, with a 30-day interval between applications;

- Conventional treatment group: participants receiving topical administration of prednisone (Predfort ${ }^{\circledR}$ eye drops, 1 drop $3 x$ daily, continuous use) and oral prednisolone $1 \mathrm{mg} / \mathrm{kg} 1 \mathrm{x}$ daily until symptom remission (ranging from 10-20 days) followed by $50 \%$ of the dose for another 5 days.

Mesenchymal Stem Cells and Transplantation

The MSC were supplied by a private laboratory that complies with the standards of the International Society for Cellular Therapy (ISCT) (Regenera Stem Cells Laboratory, São Paulo, Brazil). Briefly, as are described in Bittencourt et al. (2016), the steps involved in the production of MSC are: collection of adipose tissue from the peri-ovarian region of healthy dogs from 6 months to 2 years of age, during the elective castration procedure in Regenera-accredited clinics. The fragment is placed in vials containing transport medium to maintain the integrity of the material and sent immediately to the company to begin processing. Donor blood samples are also collected, which are sent to third-party laboratories for tests that attest to the quality of the donated material. After that the adipose tissue sample is processed into laminar flows and maintained in incubators at $37^{\circ} \mathrm{C}$ and $5 \% \mathrm{CO}_{2}$ for approximately 5 to 7 days, so that the first cells can migrate from the tissue. When the cells reach the ideal confluency, they are transferred to a new flask with more space for growth and so on until sufficient freezing is achieved. During the expansion process, the quality of the obtained cells is analyzed, as well as microbiological analyzes to evaluate the presence of contaminating agents such as bacteria, fungi, and mycoplasma. When the adipose tissue has already been sufficiently expanded, the cells are collected, counted and cryopreserved in a freezer at $-80^{\circ} \mathrm{C}$ in duly labeled aliquots containing a bar code, which enables the traceability of the sample.

For clinical use cryopreserved MSCs are supplied packed in dry ice along with a kit for three sequential washing. The result is a cell button at the bottom of the test tube with one million MSCs that were finally resuspended in $0.2 \mathrm{ml}$ of sterile saline solution (0.9\%) (Eurofarma, São Paulo, SP, Brazil) and injected in the subconjunctival and lower lateral perilimbal region using a $25 \times 0.7 \mathrm{~mm}$ needle in a $1 \mathrm{ml}$ syringe (Figures 4 and 5). For the MSC transplantation procedure, the animals were sedated with propofol (5 mg/kg, Ourofino, Cravinhos, SP, Brazil) and tramadol (2 mg/kg, União Química, São Paulo, SP, Brazil) and then, both eyes and the surrounding skin were prepared aseptically. The second transplant, with the same technical rigor, was performed thirty days after the first one.

\subsection{Groups Comparison regarding ocular surface:}

Comparisons were performed regarding affected and pigmented areas. A baseline comparison was made intergroups. Posteriorly, an evaluation was performed intragroup at baseline 30 and 110 days as well as for intergroups considering the same parameters and visits

\subsection{Statistical analysis}

The data were made using a free software on the internet, qualified for image analysis (ImageJ -http://imagej.nih.gov/ij/). The readings of changes, in the ocular surface in relation to the percentage of the area of vascularization and the area of pigmentation performed by the examiners were compared to verify the consistency between them and to evaluate the statistical similarity of both groups at the baseline data. For statistical analyses, the non-parametric data of the clinical 
evolution of the areas of vascularization and pigmentation were performed by the Wilcoxon test within each group. The Mann-Whitney test was used for comparisons between the groups. In all analysis, $p<0.05$ was set as statistical significance

\section{Abbreviations}

CSK: Chronic superficial keratitis; HGF: growth factor of hepatocytes; IDO: Indoleamine dioxygenase; IFN-ү: Interferon-ү; IL- : interleukins; LE: Left eye; MHC: Major histocompatibilty complex; MSC: mesenchymal stem cells; PGE2- Prostaglandin E2; RE: Right eye; TGF- $\beta$ : Transforming grouth factor $\beta$; Th- :Helper-T lymphocyte;

\section{Declarations}

\section{Ethics approval and consent to participate}

All procedures with animals were conducted according to the guidelines approved by the Ethics Committee of Care and Animal Use of the State University of Campinas (UNICAMP), Brazil, under protocol number 3636-1. The owners of the animals signed the free and informed consent form.

\section{Consent to publish}

Not applicable.

\section{Availability of data and materials}

All data generated or analyzed during this study are included in this published article and its supplementary information file.

The dissertation is available at the link:

http://repositorio.unicamp.br/jspui/handle/REPOSIP/332691

\section{Competing interests}

The authors declare that they have no competing interests.

\section{Funding}

This research did not receive any specific grant from funding agencies in the public, commercial, or not-for-profit sectors.

\section{Authors' contributions}

ALP: conceived the study, carried out the cell implantation, participated in clinical evaluation and post-implantation monitoring and drafted the manuscript and responded to the reviewers;

MKWB: participated in clinical evaluation and post-implantation monitoring;

MAB developed the cell implantation protocol and participated in its coordination; 
RM participated in clinical evaluation and post-implantation monitoring;

JFPM developed the cell implantation protocol;

BM carried out the cell differentiation experiments and drafted the manuscript;

FMF: participated in the design of the study and helped to draft the manuscript;

JPCV: conceived the study, participated in its coordination and helped to draft the manuscript

All authors read and approved the final version of the manuscript.

\section{Acknowledgements}

This work used material from the Regenera Stem Cell Laboratory, São Paulo, Brazil, which meets the standards of the International Society for Cellular Therapy (ISCT).

\section{References}

1. Überreiter O. Eine besondere Keratitisform (Keratitis superficialis chronica) beim Hunde. Wien Tieraerztl Monatsschr. 1961;2:65-77.

2. Balicki I. Clinical study on the application of tacrolimus and DMSO in the treatment of chronic superficial keratitis in dogs. Pol J Vet Sci. 2012;15(4):667-76.

3. Williams DL. Major histocompatibility class II expression in the normal canine cornea and in canine chronic superficial keratitis. Vet Ophthalmol. 2005;8(6):395-400.

4. Drahovska Z, Balicki I, Trbolova A, Mihalova M, Holickova M. A retrospective study of the occurrence of Chronic Superficial Keratitis in 308 German Shepherd dogs: 1999-2010. Pol J Vet Sci. 2014;17(3):543-6.

5. Espana EM, Di Pascuale MA, He H, Kawakita T, Raju VK, Liu CY, et al. Characterization of corneal pannus removed from patients with total limbal stem cell deficiency. Investig Ophthalmol Vis Sci. 2004;45(9):2961-6.

6. Slatter DH. Cornea and Esclera. In: Fundamentals of Veterinary Ophthalmology. São Paulo: Roca; 2005. pp. 283-338.

7. Williams DL. Histological and immunohistochemical evaluation of canine chronic superficial keratitis. Res Vet Sci. 1999;67(2):191-5.

8. Balicki I, Sobczyńska-Rak A. Serum vascular endothelial growth factor concentration in dogs diagnosed with chronic superficial keratitis. Acta Vet Hung. 2014;62(1):22-32.

9. BEDFORD PGC, LONGSTAFFE JA. Corneal pannus (chronic superficial keratitis) in the German Shepherd Dog. J Small Anim Pract. 1979;20(1):41-56.

10. Jokinen P, Rusanen EM, Kennedy LJ, Lohi H. MHC class II risk haplotype associated with Canine chronic superficial keratitis in German Shepherd dogs. Vet Immunol Immunopathol. 2011;140(1-2):37-41.

11. Barrientos LS, Zapata G, Crespi JA, Posik DM, Díaz S, It V, et al. A study of the association between chronic superficial keratitis and polymorphisms in the upstream regulatory regions of DLA-DRB1, DLA-DQB1 and DLA-DQA1. Vet Immunol Immunopathol. 2013;156(3-4):205-10.

12. Cheng S, Wigney D, Haase B, Wade CM. Inheritance of chronic superficial keratitis in Australian Greyhounds. Anim Genet. 2016;47(5):629.

13. Denk N, Fritsche J, Reese S. The effect of UV-blocking contact lenses as a therapy for canine chronic superficial keratitis. Vet Ophthalmol. 2011;14(3):186-94.

Page $12 / 18$ 
14. Allgoewer I, Hoecht S. Radiotherapy for canine chronic superficial keratitis using soft X-rays (15 kV). Vet Ophthalmol. 2010;13(1):20-5.

15. Williams DL, Hoey AJ, Smitherman P. Comparison of topical cyclosporin and dexamethasone for the treatment of chronic superficial keratitis in dogs. Vet Rec [Internet]. 1995 Dec 16 [cited 2020 Apr 24];137(25):635-9. Available from: http://www.ncbi.nlm.nih.gov/pubmed/8693674.

16. Nell B, Walde I, Billich A, Vit P, Meingassner JG. The effect of topical pimecrolimus on keratoconjunctivitis sicca and chronic superficial keratitis in dogs: Results from an exploratory study. Vet Ophthalmol. 2005;8(1):39-46.

17. Balicki I, Trbolova A. Clinical evaluation of tacrolimus eye drops for chronic superficial keratitis treatment in dogs. Bull Vet Inst Pulawy. 2010;54(2):251-8.

18. Caplan Al. Mesenchymal stem cells. J Orthop Res. 1991;9(5):641-50.

19. Friedenstein AJ. Precursor Cells of Mechanocytes. Int Rev Cytol. 1976;47(C):327-59.

20. Kerkis I, Kerkis A, Dozortsev D, Stukart-Parsons GC, Gomes Massironi SM, Pereira LV, et al. Isolation and characterization of a population of immature dental pulp stem cells expressing OCT-4 and other embryonic stem cell markers. Cells Tissues Organs. 2007 Apr;184(3-4):105-16.

21. Ricardo JR, da S, Gomes. JAP. Uso de células-tronco cultivadas ex vivo na reconstrução da superfície ocular. Arq Bras Oftalmol. 2010;73(6):541-7.

22. Aramburú Junior M, Luis Pippi A. Células-Tronco Mesenquimais Adultas: Características E Aplicações Experimentais Em Animais. Veterinária e Zootec. 2013;20(0):49-59.

23. Villatoro AJ, Fernández V, Claros S, Rico-Llanos GA, Becerra J, Andrades JA. Use of adipose-derived mesenchymal stem cells in keratoconjunctivitis sicca in a canine model. Biomed Res Int. 2015;2015.

24. Bittencourt MKW, Barros MA, Martins JFP, Vasconcellos JPC, Morais BP, Pompeia C, et al. Allogeneic Mesenchymal Stem Cell Transplantation in Dogs with Keratoconjunctivitis Sicca. Cell Med. 2016;8(3):63-77.

25. Villatoro AJ, Claros S, Fernández V, Alcoholado C, Fariñas F, Moreno A, et al. Safety and efficacy of the mesenchymal stem cell in feline eosinophilic keratitis treatment. BMC Vet Res. 2018;14(1):1-13.

26. Yao L, Li Z, Su W, ru, Li Y, ping, Lin M li, Zhang W xin, et al. Role of mesenchymal stem cells on cornea wound healing induced by acute alkali burn. PLoS One. 2012;7(2).

27. Williams AR, Hare JM. Mesenchymal stem cells: Biology, pathophysiology, translational findings, and therapeutic implications for cardiac disease. Vol. 109, Circulation Research. Circ Res; 2011. p. 923-40.

28. Lee MJ, Ko AY, Ko JH, Lee HJ, Kim MK, Wee WR, et al. Mesenchymal stem/stromal cells protect the ocular surface by suppressing inflammation in an experimental dry eye. Mol Ther. 2015;23(1):139-46.

29. Nagaya N, Kangawa K, Itoh T, Iwase T, Murakami S, Miyahara Y, et al. Transplantation of mesenchymal stem cells improves cardiac function in a rat model of dilated cardiomyopathy. Circulation. 2005;112(8):1128-35.

30. Caplan Al, Dennis JE. Mesenchymal stem cells as trophic mediators. Journal of Cellular Biochemistry J Cell Biochem. 2006;Vol. 98:1076-84.

31. Araujo F. The immunomodulation of CD4 + and CD8 + T cells induced by mesenchymal stem cell responses. Faculdade de Medicina de Ribeirão Preto; 2010.

32. $10.1038 / \mathrm{mi} .2010 .26$

Stern ME, Schaumburg CS, Dana R, Calonge M, Niederkorn JY, Pflugfelder SC. Autoimmunity at the ocular surface: Pathogenesis and regulation. Mucosal Immunol [Internet]. 2010;3(5):425-42. Available from: http://dx.doi.org/10.1038/mi.2010.26.

33. Hurn S, Mc Cowan C, Turner A. Oral doxycycline, niacinamide and prednisolone used to treat bilateral nodular granulomatous conjunctivitis of the third eyelid in an Australian Kelpie dog. Vet Ophthalmol. 2005;8(5):349-52.

34. Hartley C. Treatment of corneal ulcers. What are the medical options? J Feline Med Surg. 2010;12(5):384-97. 
35. Breaux CB, Sandmeyer LS, Grahn BH. Imimmohistochemical investigation of canine episcleritis. Vet Ophthalmol. 2007;10(3):168-72.

36. Jokinen P. Identifying Genetic Risk Factors in Canine Autoimmune Disorders. Molecular Medicine. 2011.

37. Ledbetter EC, Marfurt CF, Dubielzig RR. Metaherpetic corneal disease in a dog associated with partial limbal stem cell deficiency and neurotrophic keratitis. Vet Ophthalmol. 2013;16(4):282-8.

38. Liberg D, Smale ST, Merkenschlager M. Upstream of Ikaros. Trends Immunol. 2003;24(11):567-70.

39. Machado C, de V, Telles, PD da S, Nascimento ILO. Immunological characteristics of mesenchymal stem cells. Rev bras hematol hemoter. 2013;62-7.

40. Sanchez RF, Daniels JT. Mini-Review: Limbal Stem Cells Deficiency in Companion Animals: Time to Give Something Back? Curr Eye Res. 2016;41(4):425-32.

\section{Figures}

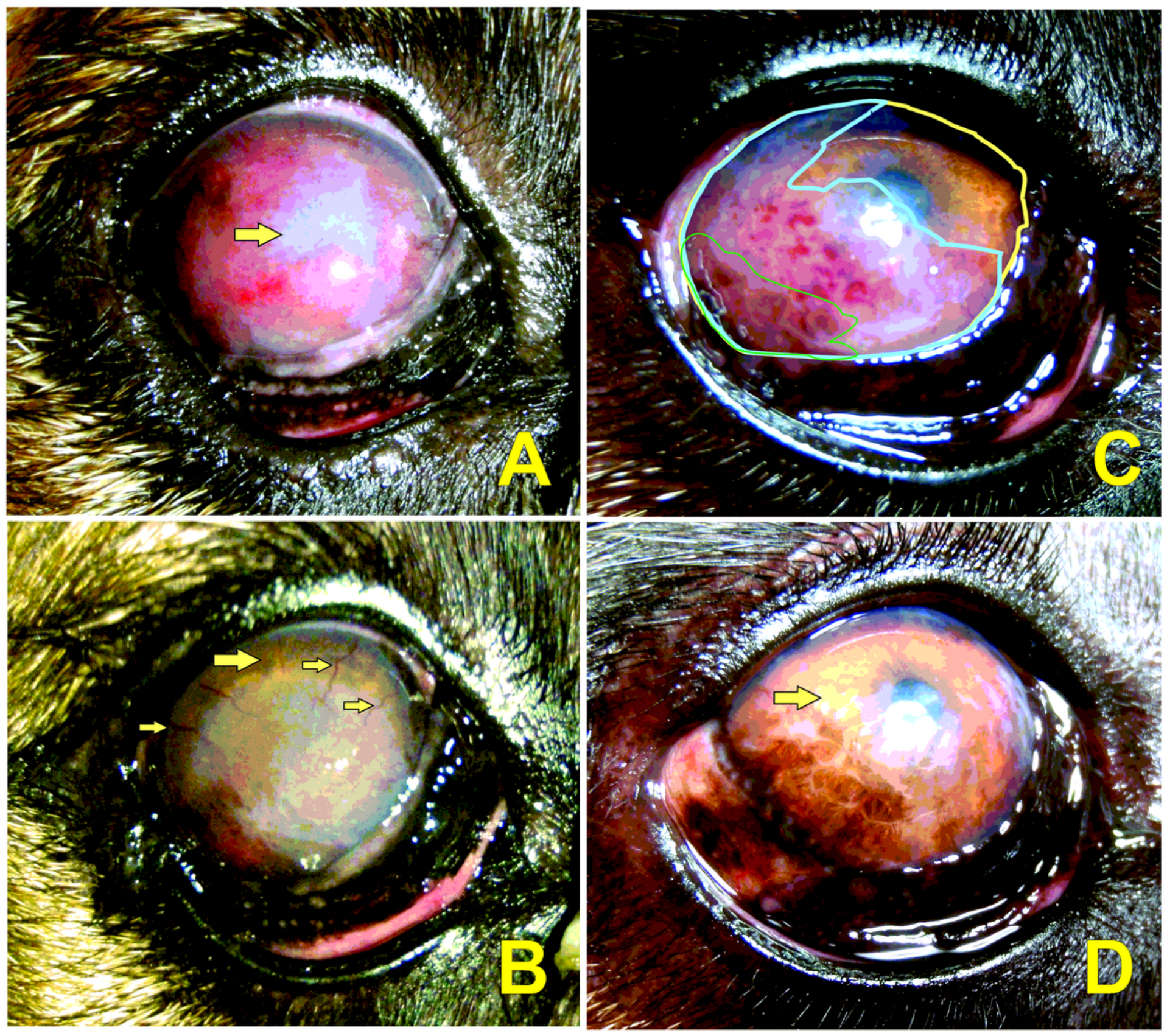




\section{Figure 1}

Participants of the experimental group. A and B present the right eye of participant \#1 on days 0 and 110 respectively. C and $D$ present the right eye of participant \#2 at days 0 and 110 respectively. In $A$ the neovascularization blocked anterior chamber visibility along with poor visual ability behavior evidenced by the negative result in the menace response test (arrow). The In B, neovascularization decline to the point where only a small number of blood vessels were visible (small arrows), the periphery of the cornea became more transparent (large arrows) and visual behavior was clinically normal. In $\mathrm{C}$, the disease stage was just the lateral portion of the cornea affected. As a model, the color lines represent the measurements analyzed: yellow represents the total area of the cornea, blue is the affected area and green the pigmented area. In $\mathrm{D}$, at the end of the follow-up, the cornea had no blood vessel with cornea becoming clear (arrow) but the pigmentation clinically occupied the same area as the initial blood vessels.
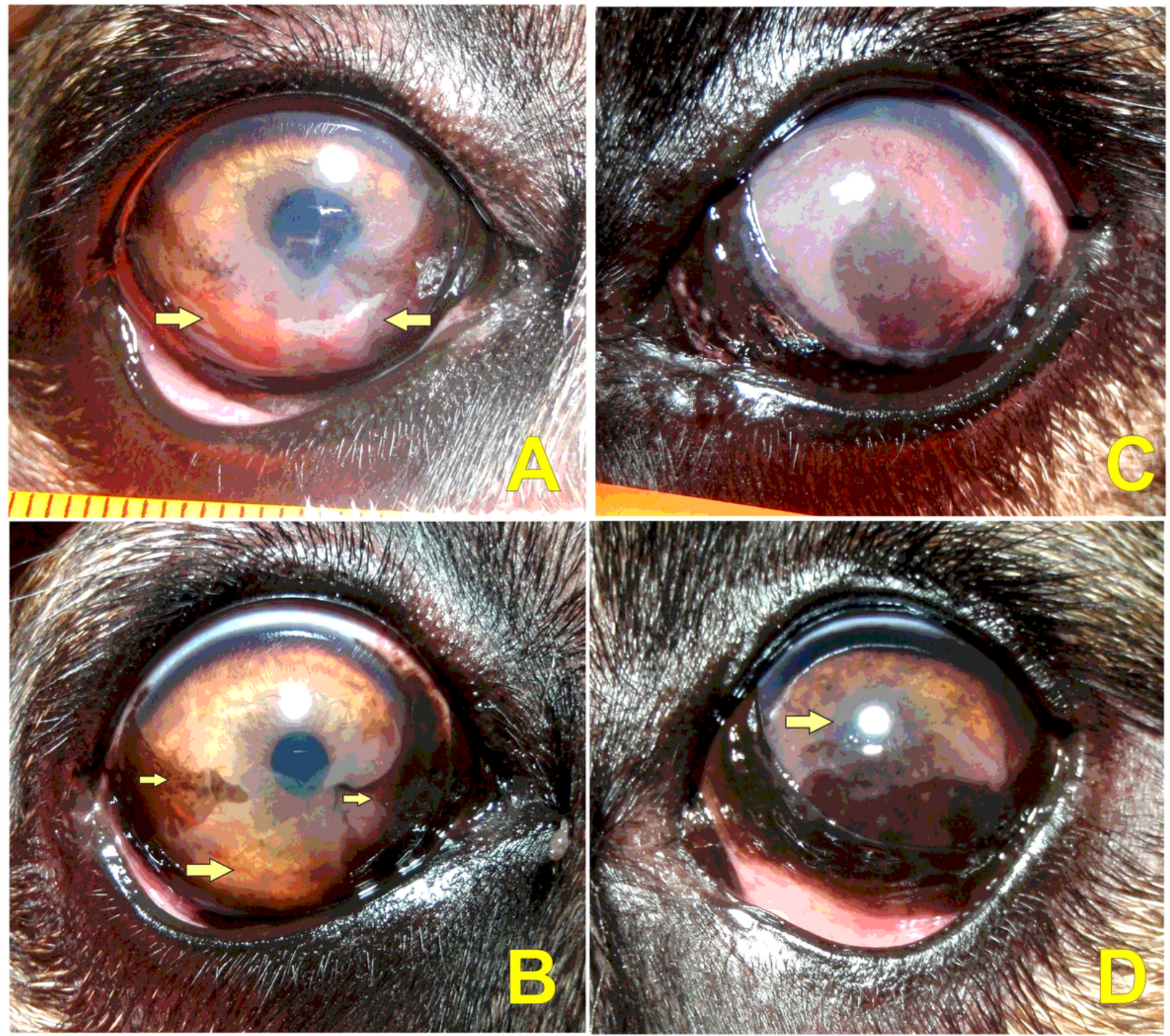

Figure 2 
Participants of the conventional group. A and B present the right eye of participant \#6 on days 0 and 110 respectively. C and $D$ present the left eye of participant \#7 at days 0 and 110 respectively. In $A$, the disease at the initial stage with only two lower quadrants affected (arrows). In B, the neovascularized area had regressed without became pigmented (large arrow) and the initial pigmented area had established peripherally (small arrows). In C, the disease was in a more advanced stage with a chronic pattern due to the presence of an extensive visible pigmentation associated with the neovascularization. In D, the cornea showed the dorsal area cleaned (arrow) and the pigmented area had regressed in central area allowing visual menace response and visual capability
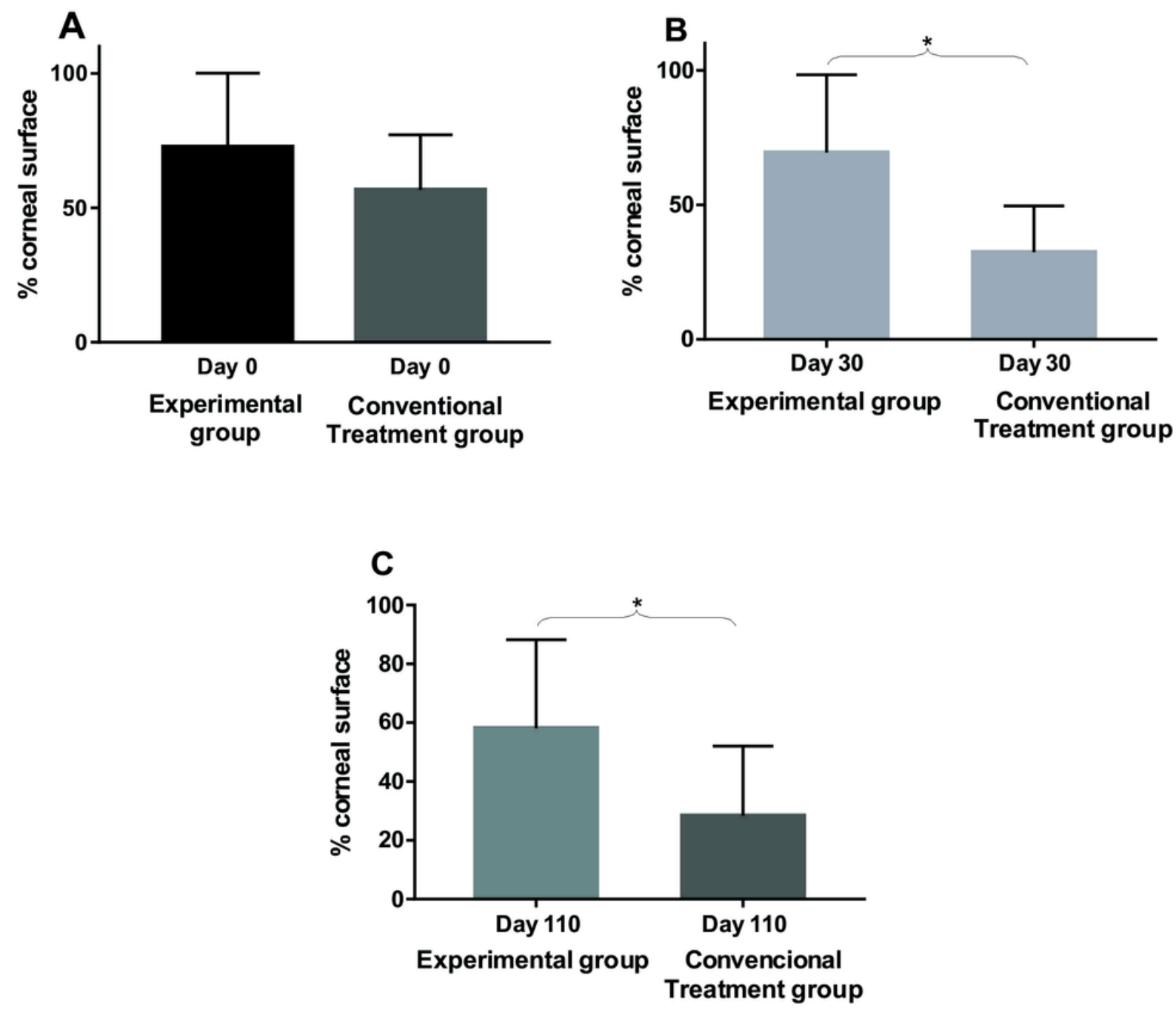

Figure 3

Group Comparisons. A. Comparison between groups on day 0 , with no statistical difference between their values $(p=0.28)$. B. Comparison between groups on day 30 , with statistical difference $\left.{ }^{*}\right)$ among their values $(p=0.04)$. C. Comparison between groups on day 110 , with statistical difference $\left.{ }^{*}\right)$ among their values $(p=0.03)$. Columns represent mean and standard deviation, $\mathrm{n}=8$. 

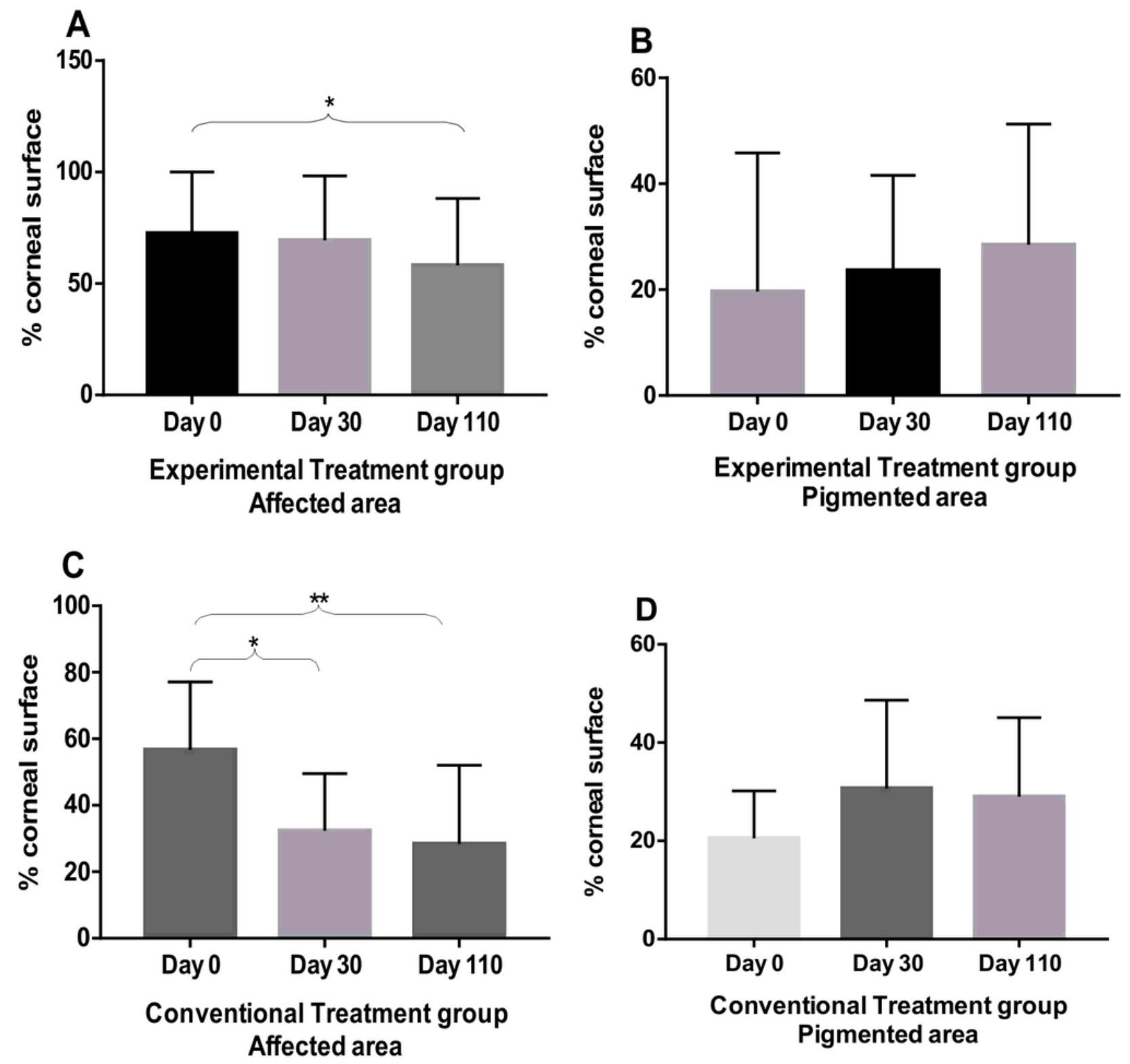

Figure 4

Evaluation progress. A Experimental group: affected areas. Comparison between follow-up days. There was no statistical difference between day 0 and day $30(p=0.25)$ and with statistical difference $\left.{ }^{*}\right)$ between day 0 and day $110(p=0.008)$.B Experimental group: pigmented areas. Comparison between follow-up days. No statistical difference between day 0, 30 and day 110 ( $p=0.25)$. C Conventional Treatment Group: affected areas. Comparison between follow-up days. With statistical difference $\left(^{\star}\right)$ between day 0 and day $30(p=0.03)$ and with statistical difference $(* *)$ between day 0 and day 110 $(p=0.04)$. D Group Conventional treatment: pigmented areas. Comparison between follow-up days. No statistical difference between day 0 and day $110(p=0.4)$. Columns represent the mean and standard deviation, $n=8$.

\section{Supplementary Files}

This is a list of supplementary files associated with this preprint. Click to download. 
- PereiraALARRIVEChecklist.pdf

- PereiraALDatabaseStemcellChronicSuperfKeratitis.xIsx 